\title{
Global Health in Medical Education
}

\author{
Camille Z. Bentley ${ }^{1}$ (1)
}

Accepted: 3 September 2021 / Published online: 16 September 2021

(c) International Association of Medical Science Educators 2021

\begin{abstract}
In this age of increased global connectivity, global health education is more important than ever. While numerous residency programs include global programs, less are offered during medical school. Many of these are during clinical years with little training prior to participating in an international externship. The Global Medicine Track (GMT) at Rocky Vista University is a 3.5-year formal osteopathic program aimed at training culturally competent physicians to more effectively serve global and underserved communities. Major goals include intensive didactic training and experiential clinical activities. Ongoing evaluations and assessments support the topics covered as relevant for today's Global Health needs.
\end{abstract}

When reviewing the literature on global health in medical education, it appears that as early as $1982,6.4 \%$ medical students were already performing immersive international clinical training rotations, with the purpose of seeing diseases and cultures not experienced here in the USA [1]. I was introduced to Global Health $(\mathrm{GH})$ about 20 years ago, when I not only volunteered, but organized a group of students and health care providers to travel to Guatemala with the help of DOCARE international to provide much needed healthcare to the indigenous Mayan living in the Lake Atitlan area. Despite being quite naïve when it came to global health, the people there captured my heart, and I continue to travel there annually to provide care. After that very first trip, I realized that if I was to continue with this type of work, I needed to immerse myself, learning as much as I could about global health, to better serve others. I even went on to get my MPH degree.

In my 20 years, I have seen a dramatic growth of $\mathrm{GH}$ as a discipline of study in medical education. Whether it be interest groups, pathways, tracks, or just the increase in student travel internationally as part of the clinical training, or to just volunteer themselves in medical capacity, this growth is quite evident in both medical schools and residencies.

Both student interest in Global Medicine and the expansion of Global Health as an academic discipline have

Camille Z. Bentley

cbentley@rvu.edu

1 Family Medicine, Tracks and Special Programs Department, Rocky Vista University College of Osteopathic Medicine, Parker, CO, USA continued to increase over the last 40 years [2-5]. Many schools are and continue to establish or strengthen GHrelated programs. The increasing number of residency programs now offers Global Health Tracks/Pathway within or across specialties to accommodate trainees' continued interest and desire for clinical opportunities in the GH arena. More students are participating in projects and programs related to addressing health disparities outside of their home countries, and many of them inquire about such global opportunities during their medical school interviews. This results in more American students entering medical schools and residencies with a myriad of $\mathrm{GH}$ experiences.

There is a renewed interest in the field of public health, especially in light of the recent pandemic. "The number of medical school applicants is up $18 \%$ this year over last year, according to the Association of American Medical Colleges, or AAMC, probably driven by the example of medical workers and public health figures such as Dr. Anthony Fauci."

How significant is the impact of Global Medicine education now with the advent of the COVID-19 pandemic and the obvious connectivity that exists among all nations and peoples in the world? If this pandemic does not highlight the need for globally competent health care physicians among the myriad of other global systems and workers, I am not sure what would!

Regardless of the sponsoring organization, location, and type of outreach, being able to provide patients with personal healthcare outside of the USA is what seems to attract many to Global Health, but in reality, this is a very small component of the field of Global Health and our Global Med program at RVU. It is certainly for many students the 
culmination of hours of learning and preparing to provide quality care for the diverse populations we serve living in resource-poor countries. Young adults today, specifically those in HS and college, are well connected to the world via current technologies. Many have friends, acquaintances, and even family living in other parts of the world. Students in medical school are not any different.

So, each October, when my colleague and I, as co-directors of the RVUCOM Global Medicine Track, start the process of putting together the next class, there is a great deal of thought as to what attributes we would like to see in the students we accept for this program.

A Merck Manuals Survey was conducted at the American Medical Student Association (AMSA) Annual Convention in March of 2018 to uncover students' motivations and aspirations in Global Health [7]. Even though the survey was administered to only 130 students, they did include both medical and pre-medical students representing domestic and international universities and programs. I feel what they found is typical of the students applying to med school and our track. When asked about their top motivation for getting involved in global health, a majority of students (59 percent) said they want to provide health care where it is needed most. Fourteen percent said they have an interest in studying infectious diseases and conditions, and $10 \%$ want to have cross-cultural experiences. Many students intend to turn this inspiration into action by experiencing healthcare practices from around the world. Students who plan to get involved in global health $64 \%$ said they are planning to take shortterm service trips to "underdeveloped" areas, 38\% engage in research to solve global healthcare issues, 33\% pursue long-term clinical work in underdeveloped areas, $22 \%$ work with a non-governmental organization (NGO), and $18 \%$ get involved in global health policy and diplomacy work.

So, regardless of our diverse backgrounds, during our interviews, my colleague and I have conversations on these topics as well as the students' past exposure to other cultures and languages, current problems in healthcare and their thoughts on why these exist, and perhaps even some thoughts on possible solutions. Last but not least, what have they already accomplished and plan to do as physicians in the GH arena.

Some come well prepared, and others get the chance to reflect for the first time. Then, regardless of the students' backgrounds, we put together a class of lifelong learners that hopefully someday will make their contribution to promote health and wellbeing for others around the globe.

There are both perceived and documented benefits of international clinical training experiences for medical students and for physicians volunteering in this capacity $[6,9]$. Traveling to other countries and experiencing new cultures and languages has played a major role in assisting students to become more culturally sensitive and humble when working with patients different from themselves. It is believed being able to explore the global and different views of healthcare assists students in making an informed choice about one's profession, and it helps to cement their commitment toward medicine for underserved populations. After experiencing other health care systems (esp. dealing with low cost and lack of resource allocations), students can become stronger clinicians, and/or better administrators in our own health care arena.

It is also felt that participation in these experiences increases the likelihood to volunteer abroad, work with underserved pops, pursue a career in public health or primary care, obtain a public health degree, or volunteer internationally in the future. Finally, participation in international health electives has been shown to provide expanded medical knowledge, improved physical examination skills, reduced dependence on laboratory and/or radiologic tests, and enhanced professionalism, thus producing more competent physicians.

\section{Global Medicine Track, RVUCOM}

In 2011, RVUCOM established the Department of Tracks and Special Programs. This is a department dedicated to providing interested students additional curricula in various disciplines focusing on the needs and future of medical practice in this country. Currently, we offer multiple courses covering diverse topics in healthcare on both campuses, in addition to the core "These are all optional additional, credited courses that complement the required core curriculum."

The Global Medicine Track (GMT) is aimed at training culturally sensitive and clinically competent physicians to more effectively serve global and underserved communities, both home and abroad.

The GMT is a 3.5-year formally assessed osteopathic training program (part of transcript) that begins in January of the first year. Students accepted into this track spend about $100 \mathrm{~h}$ over 3 semesters, and then must complete a minimum of 3 global health-related externships/activities during their clinical years to graduate with distinction of participation.

Each semester focuses on specific themes including but not limited to public heath, health systems, ethics, disease burden, and emerging and reemerging diseases, all from a global health perspective. Students are also encouraged to participate in some form of a research project during their tenure at the COM. They must maintain $80 \%$ (PASS) or $91 \%$ (HONOR) to participate while maintaining passing grades in core curriculum. All of tracks work on a point system.

We use multiple forms of pedagogy including open, learner centered, and case based. Over the past few years, we have moved to a "flipped classroom" approach, which has worked really well in keeping our students engaged. There is great flexibility for the student to become exposed 
and review the information on their own time schedule. When they arrive in class, prepared students can start engaging in higher-level learning and critical thinking. We work as facilitators, something even I have had to learn and become more comfortable with, over the past few years. We have found this to be a really powerful way to learn material and content correctly, while being more efficient so we can actually cover a great deal of information face to face or now virtually, in less time. Time is spent on advising students who present topics of their choice and guiding ensuing discussion on those topics. We are there to support them in their research, help them define or hone their topic to fit in the amount of time allowed for the presentation, and to assist them with improving their presentation skills while using any type of technology to which they have access and choose to use. Most use a PowerPoint as their base, with embedded videos, games, or case-based scenarios for discussion by the class as a whole or in small groups. Many students even provide pre-class activities for the other students to complete. They are extremely innovative and engaged in this form of adult learning. Finally, we include appropriate hands-on experiences which are listed later.

The course for the last 3 years is co-directed by two faculty members, myself as a physician and a biomedical scientist. Together, we have 46 years' experience teaching in higher education. Having two facilitators bringing different perspectives to the class has been a benefit to the students encouraging them to consider multiple viewpoints when assessing their own ideas on the topic.

The highlights of this course are the hands-on activities we provide for our students in the GMT. These activities are not provided in the core (required by all students) curriculum. Assessments are involved with all these activities; students are usually provided with pre-lab review resources, training reviews by faculty; and each is followed up with a written self- reflective/evaluative assignment. Students are expected to complete the following activities/cases following the outcomes assessed for each activity. They include 3D virtual reality, SP encounters involving working with an interpreter, working alone with a Spanish-speaking patient, and working via telemedicine and airline emergencies. We also include labs taking a closer look at feet, mouths, eyes, wound, and abscess care. All this eventually culminates into participating in Clinical International Externships with RVU or other approved organizations.

We must adhere to all the COM requirements for proper documentation related to National Accreditation and AOA/ residency standards. Remember though, we are curricular in nature with the necessary syllabi, student learning outcomes, assessments, and course evaluations. The actual course content is not prescribed, so we are allowed to be flexible and innovative with both content and delivery.

\section{Pandemic Arrival, March 2020}

With the onset of the pandemic, most of the curriculum at Rocky Vista University moved to a virtual platform. Since our track programs are not part of the core curriculum, we were asked to go $100 \%$ virtual. This was a challenge for many in my department, because of the emphasis of handson activities for many of the classes. In the Global Medicine Track, our main focus became how to keep the students engaged and feeling that they belonged to a special group. First and foremost, we took a few minutes at the beginning of each class to talk about staying connected and caring for one another. As for the coursework, we continued teaching in both a student-centered and flipped classroom. We also encouraged students to present topics they felt relevant to themselves in global health today rather than being assigned topics of our choosing. By student request, we added a 5-10min current events report, one presented by a student at the beginning of each class. Because we do have much cultural diversity of students in this class, many would discuss what was happening with the COVID-19 pandemic and vaccine situation in a country with which they were personally connected. Despite the restrictions to in-person learning due to the pandemic, my colleague and I were still able to complete all of the following presentations by faculty and students via Zoom, as well as most of the hands-on activities.

\section{Global Local}

One area the pandemic really affected was being able to travel internationally for clinical immersion with diverse populations. Travel was halted in March 2020; however, we plan to resume travel in January 2022. This pandemic reinforced the concept of going "glocal". In reality, we do not actually have to travel very far to have the experience of providing appropriate health care to those who are poor, indigenous, and/or culturally diverse from ourselves. Here in the USA, and especially in Denver, we are surrounded by diverse populations from all over the world, regardless of economy, geography, or culture [8].

\section{Future of Global Health in Medical Education}

Concepts of Public Health, Epidemiology, Cultural Humility, emerging and re-emerging diseases, and ethical issues surrounding equity and access to care have already been incorporated into the curricula of many medical school programs. For many schools, the concept of going glocal I believe will continue to become popular as it would be much easier and less costly for schools to incorporate into student training, whether curricular or extracurricular. Our country is very diverse culturally, geographically, and economically, 
so students really do not have to go overseas to get the experiences of working with diverse populations. As this occurs, Global Medicine programs such as ours will continue to expand and evolve. The advantage of having a small group of 20 students vs groups of 150 or more allows for more interaction and use of different types of activities and allows us to go where others cannot.

In 2020, the World Health Organization released a list of 13 urgent health challenges the world will face over the next decade. These issues are a good source of topics for Global Med classes.

WHO said the challenges included on the list "demand a response from more than just the health sector," adding, "governments, communities, and international agencies must work together" to address these "critical" issues. Even more reason why we need to educate physicians to work in the GH arena.

The ability to work in the global arena continues to grow, for physicians at all levels, whether new to the field or near retirement. As we continue to expand in technology, especially travel and communication, countries must work together to fight disease and insure the health and wellbeing of all citizens of the world. With continued advances in communication and travel, the world will continue to become smaller, and the health and wellbeing of people living throughout the world will affect all of us.

I believe that all primary care health care workers should be trained to think from a public health/global health perspective, providing the most appropriate care for their patients, regardless of location. We all should be culturally open to differences and empathic to the geographical and socioeconomic context which define our patients' lives. Having a global health program in medical education is a significant way to train our future physicians.

\section{Declarations}

Ethics Approval and Consent to Participate Both ethical approval and informed consent are not applicable.

Competing Interests The author declares no competing interests.

\section{References}

1. Heck JE, Pust R. A national consensus on the essential internationalhealth curriculum for medical schools. Acad Med. 1993;68(8):596-8.

2. Drain P, Holmes KK, Skeff KM, Hall TL, Gardner P. Global health training and international clinical rotations during residency: current status, needs, and opportunities. Acad Med. 2009;84(3):320 5. https://doi.org/10.1097/ACM.0b013e3181970a37.

3. Hau, DK et al. Global health training among US residency specialties: a systematic literature review. https://doi.org/10.1080/10872 981.2016.1270020.

4. Kerry, VB et al. US medical specialty of global health training and the global burden of disease. www.jogh.org. https://doi.org/ 10.7189/jogh.03.020406.

5. Khan OA, Guerrant R, Sanders J et al. Global health education in U.S. Medical schools. BMC Med Educ. 2013;13(3). https://doi. org/10.1186/1472-6920-13-3.

6. Eckhert NL. Getting the most out of medical students' global health experience. Ann Fam Med. 2006;4(Suppl 1):S38-9. https:// doi.org/10.1370/afm.563.

7. Merck Manuals Survey: medical students seek global health experiences to enhance their education. 2018. www.merckmanuals.com.

8. Rowthorn, V. A review: global/local: what does it mean for global health educators and how do we do it? Ann Glob Health. 2015;81(5). https://doi.org/10.1016/j.aogh.2015.12.001.

9. Shannon, SC. A Look at Global Health through the Osteopathic Lens (aacom.org). 2016.

Publisher's Note Springer Nature remains neutral with regard to jurisdictional claims in published maps and institutional affiliations. 